\title{
Ascaris lumbricoides causing acute abdomen: a case report
}

M.Z.M. Abdellatif, ${ }^{\text {U.S. Belal, }}$ 'E.H. Abdel-Hafeez, ${ }^{7}$ A.M. Atiya ${ }^{2}$ and K. Norose ${ }^{3}$

\section{Introduction}

Ascaris lumbricoides is one of the most well-known helminthic parasites affecting humans, and ascariasis remains common with $>1.2$ billion infections globally [1]. It is endemic in the Middle East and South America especially in under-developed countries where poor sanitation, the most important risk factor for infection [2], is common [3]. A. lumbricoides infection occurs in all age groups but more commonly in preschool children [4]. Although ascariasis cases are usually asymptomatic, infection leads to malnutrition in children and causes about 3000-60 000 deaths every year, usually as a result of intestinal obstruction [5-7].

The most common causes of acute abdomen are acute appendicitis, acute peptic ulcer, acute cholecystitis, acute pancreatitis, intestinal obstruction, acute peritonitis and acute pyelonephritis [8]. One cause of intestinal obstruction by parasites is A. lumbricoides infection. The most commonly known serious and lethal complication of A. lumbricoides infection is intestinal obstruction, caused by an aggregated mass of $A$. lumbricoides worms, which may develop acutely or subacutely [9]. Early diagnosis of intestinal obstruction caused by A. lumbricoides using ultrasonography is very useful to avoid its serious and lethal complications [10]. Emergency surgical treatment may be necessary in acute intestinal obstruction in which the mass of the parasite obstructs the intestinal lumen or intestinal obstruction develops due to volvulus [11]. Meanwhile, subacute cases are successfully treated conservatively until spontaneous resolution $[3,12]$. The aim of this report is to present a case of bowel obstruction caused by $A$. lumbricoides as a cause of acute abdomen in one governorate in Egypt.

\section{Case report}

A 12-year-old boy from Tokh El Khail, Minia governorate, Egypt, was admitted to the emergency department of Minia University Hospital, Minia, Egypt, on 10 March 2012, with acute colicky periumbilical abdominal pain not referred to other sites, vomiting and constipation for 3 days.

On physical examination he had pallor and appeared poorly nourished. His oral temperature was $37.5^{\circ} \mathrm{C}$, and blood pressure was $100 / 60 \mathrm{mmHg}$. Pulse was regular with a rate of 120 beats per minute, and respiratory rate was 35 per minute. Respiratory examination revealed bilateral air entry with no added sounds but slight respiratory effort. Cardiovascular examination revealed normal S1 and S2 with no murmur, and central nervous system examination revealed no neurological deficits. Abdominal examination revealed abdominal tenderness and rigidity in the central and mid-abdomen. There was no organomegaly. Auscultation revealed a silent abdomen or minimal peristalsis.
At the time of admission, laboratory investigations were done. The red blood cell count was 3.4 to $3.8 \times 10^{6} / \mu \mathrm{L}$, and haemoglobin level was $10 \mathrm{~g} / 100$ $\mathrm{mL}$. The total leukocyte count was raised $\left(13 \times 10^{3} / \mu \mathrm{L}\right)$, and eosinophils were increased (12\%). Abdominal ultrasonography demonstrated parallel paired lines like "railway tracks" in the intestinal lumen that was suggestive of worms. The boy came from a very low socioeconomic status family. His father was a farmer and all the family helped the father, usually spending all the day working in the fields. They usually ate vegetables from the field without washing. The boy had a history of pica. A. lumbricoides infection was suspected as the patient's mother gave a history of expulsion of one very big worm from the ear shortly before coming to the hospital. Moreover, there were repeated attacks of vague abdominal pain, colic and some attacks of diarrhoea.

The boy was prepared for operation with correction of fluid and electrolyte imbalance, broad spectrum antibiotic as a prophylaxis, blood transfusion and analgesics. No antihelminthic drug was used at this stage. Intra-operative exploration through a right lower paramedian incision revealed serous collection. At laparotomy, he had multiple abnormal contents in the small intestine (Figure $1 \mathrm{~A}$ ). Milking was performed by hand to collect worms to the jejunum to facilitate their extrusion (Figure 1 B). An enterotomy was done where an incision was done in the intestine (Figure $1 \mathrm{C}$ ) 
to extract worms by sponge-holding forceps (Figures $1 \mathrm{D}$ and $\mathrm{E}$ ). The worms approximately filled a kidney tray (Figure $1 \mathrm{~F}$ ). There were total 53 worms: 30 adult males and 23 adult females. $\mathrm{He}$ had very healthy appendix. Exploration was done to exclude any other affected areas as well as to rule out any area of questionable viability in the rest of the gut. The abdomen was closed with a tubal drain within it.

The worms were sent to the laboratory of the department of parasitology at the faculty of medicine, Minia University for further identification. The worms were cylindrical in shape, pinkish in colour. Adult males measured 15 to 30 $\mathrm{cm}$ in length and 2 to $4 \mathrm{~mm}$ in diameter and their posterior end was curved with 2 spicules. Adult females measured 20 to $40 \mathrm{~cm}$ in length and 3 to $6 \mathrm{~mm}$ in diameter and their posterior end was straight. These worms were verified as A. lumbricoides from their macroscopic appearance and size and shape of eggs microscopically.

Amoxicillin/clavulanate combination $\left(\right.$ Augmentin $^{\circ}$ ) as a broad-spectrum antibiotic was given intravenously. On the 2 nd post-operative day the patient received a single dose of albendazole (400 mg). Three days post-operative stool examination revealed few Ascaris spp. eggs. He gave a history of passage of tall brown-yellow worms on the second day after taking albendazole. The child was discharged on the 4th day

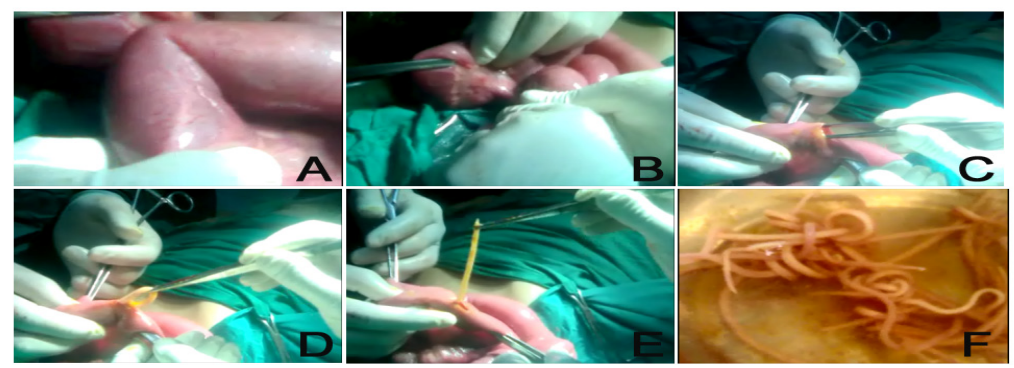

Figure 1 Steps of surgical intervention in a case of acute abdomen caused by Ascaris lumbricoides infection. (A) Abnormal multiple contents in the small intestine; (B) Milking of worms by hand; (C) Performance of enterotomy; (D, E) Extraction of worms by sponge-holding forceps; (F) Worms approximately filled a kidney tray postoperatively without complications. Stool examination had been repeated twice on the first and the second weeks post-operative. The dose of albendazole was repeated after 2 weeks following faecal examination.

\section{Discussion}

Ascariasis is a world-widely distributed parasitic infection, especially in tropical and subtropical areas where unhygienic disposal of human excreta is common. The mode of A. lumbricoides infection is by the ingestion of embryonated eggs in raw vegetables, water or soil-contaminated hands. The fertilized eggs hatch in the intestine. The released larvae penetrate the intestinal wall to reach the right side of the heart, pulmonary circulation and then to the alveoli. When the larvae are coughed up by the host, they are swallowed back into the intestine to develop into adult worms $[3,7]$.

The clinical features of A. lumbricoides infection are variable between asymptomatic and symptomatic disease depending upon the parasitic burden. The symptomatic disease is largely restricted to individuals with a high worm load [7]. The symptoms are related either to larval migration or to the adult worm intestinal stage. Pulmonary manifestations of ascariasis are due to the larval migration through the lungs. Heavy infection with $A$. lumbricoides is frequently manifested by abdominal discomfort, anorexia, nausea and diarrhoea. Heavily infected children suffer from protein malnutrition and vitamin A deficiency $[13,14]$. Adult worms cause serious clinical problems during their migration, including acute pancreatitis, acute cholecystitis, liver abscess, intestinal obstruction when present in large numbers and even perforation $[2,7]$. They may occasionally cause appendicitis and obstructive jaundice [15]. The massive gastrointestinal bleeding with ulceration, perforation and even gangrene of the bowel wall are rare, fatal complications [16]. Since it is clearly obvious from the clinical manifestations and complications of A. lumbricoides infections that $A$. lumbricoides is an important cause of acute abdomen, roundworm obstruction should be considered as the first differential diagnosis in any patient of intestinal obstruction in an A. lumbricoides-endemic area [3].

The management of the majority of cases of uncomplicated ascariasis can be done successfully with antiparasitic drugssuch as albendazole, mebendazole or pyrantel pamoate $[17,18]$. Meanwhile, complicated cases of ascariasis can be managed by surgical intervention. The type of surgery depends on the findings during laparotomy. If the bowel is viable and the obstruction is at the level of ileum, milking of the worms to the caecum can be done carefully without causing trauma to the bowel wall [19]. If the obstruction is at the level of the jejunum and if there are multiple masses, enterotomy should be done through a longitudinal incision with removal of worms by sponge-holding forceps. The incision should be closed transversally with great care to avoid contamination of the peritoneal cavity by the worms or their eggs. In cases where the intestinal wall is thin, for example in cases of volvulus, milking should not be attempted as this may cause serosal tears. Enterotomy is preferred in such cases. Breaking of the worms during milking should be avoided as this may release 
toxins. In cases presenting with bowel gangrene, perforation, or intussusception with non-viable bowel, resection with primary anastomosis may be required $[20,21]$. In our case, enterotomy was performed to remove the worms after careful milking of the worms, as multiple abnormal contents in the small intestine had been found at laparotomy.

The early diagnosis of complicated ascariasis and surgical intervention are essential to minimize high morbidity and mortality of complicated ascariasis. The awareness of ascariasis and its preventive measures should be included in all health education programmes and should be delivered to schoolchildren and their mothers to overcome the risk of infection.

\section{References}

1. De Silva NR et al. Soil-transmitted helminth infections: updating the global picture. Trends in Parasitology, 2003, 19:547-551.

2. Galzerano A, Sabatini E, Durì D. Ascaris lumbricoides infection: an unexpected cause of pancreatitis in a western Mediterranean country. Eastern Mediterranean Health Journal, 2010, 16:350-351.

3. Schulze SM et al. Acute abdomen secondary to Ascaris lumbricoides infestation of the small bowel. American Surgeon, 2005, 71:505-507.

4. Steinberg R et al. Unusual intestinal sequelae after operations for Ascaris Lumbricoides infestation. Pediatric Surgery International, 2003, 19:85-87.

5. Baba AA, Ahmad SM, Sheikh KA. Intestinal ascariasis: the commonest cause of bowel obstruction in children at a tertiary care center in Kashmir. Pediatric Surgery International, 2009, 25:1099-1102.

6. Hotez PJ et al. Rescuing the bottom billion through control of neglected tropical diseases. Lancet, 2009, 373:1570-1575.

7. Dold C, Holland CV. Ascaris and ascariasis. Microbes and Infection, 2011, 13:632-637.

8. Moradpour D, Blum HE. Acute abdominal pain. In: Siegenthaler W, ed. Differential diagnosis in internal medicine: from symptom to diagnosis. New York, Thieme, 2007:257-273.

9. Sarmast $\mathrm{AH}$ et al. Duodenal perforation with an unusual presentation: a case report. Case Reports in Infectious Diseases, 2011, 2011:512607.

10. Mehta $V$ et al. Sonographic diagnosis of Ascaris Lumbricoides infestation as a cause of intestinal obstruction. Indian Journal of Pediatrics, 2010, 77:827.

11. Bethony J et al. Soil-transmitted helminth infections: ascariasis, trichuriasis, and hookworm. Lancet, 2006, 367:1521-1532.

12. Hefny AF, Saadeldin YA, Abu-Zidan FM. Management algorithm for intestinal obstruction due to ascariasis: a case report and review of the literature. Ulusal Travma ve Acil Cerrahi Dergisi, 2009, 15:301-305.

13. Stephenson LS, Latham MC, Ottesen EA. Malnutrition and parasitic helminth infections. Parasitology, 2000, 121(Suppl.):S23S38.

14. Crompton DW, Nesheim MC. Nutritional impact of intestinal helminthiasis during the human life cycle. Annual Review of Nutrition, 2002, 22:35-59.

15. Sandouk F et al. Pancreatic-biliary ascariasis: experience of 300 cases. American Journal of Gastroenterology, 1997, 92:2264-2267.

16. Kawatra $V$ et al. Gangrene intestine caused by Ascaris lumbricoides; report of 5 cases in children. Pathology, Research and Practice, 2010, 206:292-294.

17. Tracy JW, Webster LT. Drugs used in the chemotherapy of helminthiasis. In: Hardman JG et al., eds. Goodman and Gilman's the pharmacological basis of therapeutics, 9th ed. New York, McGraw-Hill, 1996.

18. Keiser J, Utzinger J. Efficacy of current drugs against soiltransmitted helminth infections: systematic review and metaanalysis. Journal of the American Medical Association, 2008, 299:1937-1948.

19. Shiekh KA et al. Mechanical small bowel obstruction in children at a tertiary care centre in Kashmir. African Journal of Paediatric Surgery, 2010, 7:81-85.

20. Yetim I et al. Rare cause of intestinal obstruction, Ascaris lumbricoides infestation: two case reports. Cases Journal, 2009, 2:7970.

21. Mukhopadhyay B et al. Clinical appraisal of Ascaris Lumbricoides, with special reference to surgical complications. Pediatric Surgery International, 2001, 17:403-405. 